\title{
Genetically Induced Adult Oligodendrocyte Cell Death Is Associated with Poor Myelin Clearance, Reduced Remyelination, and Axonal Damage
}

\author{
Hartmut B. F. Pohl, ${ }^{1}$ Cristina Porcheri, ${ }^{1,2}$ Thomas Mueggler, ${ }^{3}$ Lukas C. Bachmann, ${ }^{1}$ Gianvito Martino, ${ }^{2}$ \\ Dieter Riethmacher, ${ }^{4}$ Robin J. M. Franklin, ${ }^{5}$ Markus Rudin, ${ }^{3,6}$ and Ueli Suter ${ }^{1}$ \\ ${ }^{1}$ Institute of Cell Biology, Department Biology, Eidgenössische Technische Hochschule (ETH) Zürich, CH-8093 Zürich, Switzerland, ${ }^{2}$ Neuroimmunology \\ Unit, DIBIT-2, Institute of Experimental Neurology, Division of Neuroscience, San Raffaele Scientific Institute, 20132 Milano, Italy, ${ }^{3}$ Institute of Biomedical \\ Engineering, Department of Information Technology and Electrical Engineering, University of Zürich and ETH Zürich, CH-8093 Zürich, Switzerland, \\ ${ }^{4}$ Human Genetics Division, Southampton University School of Medicine, Southampton S016 6YD, United Kingdom, ${ }^{5}$ Medical Research Council Cambridge \\ Centre for Stem Cell Biology and Regenerative Medicine, and Department of Veterinary Medicine, University of Cambridge, Cambridge CB3 OES, United \\ Kingdom, and ' Institute of Pharmacology and Toxicology, University of Zürich, CH-8093 Zürich, Switzerland
}

Loss of oligodendrocytes is a feature of many demyelinating diseases including multiple sclerosis. Here, we have established and characterized a novel model of genetically induced adult oligodendrocyte death. Specific primary loss of adult oligodendrocytes leads to a well defined and highly reproducible course of disease development that can be followed longitudinally by magnetic resonance imaging. Histological and ultrastructural analyses revealed progressive myelin vacuolation, in parallel to disease development that includes motor deficits, tremor, and ataxia. Myelin damage and clearance were associated with induction of oligodendrocyte precursor cell proliferation, albeit with some regional differences. Remyelination was present in the mildly affected corpus callosum. Consequences of acutely induced cell death of adult oligodendrocytes included secondary axonal damage. Microglia were activated in affected areas but without significant influx of B-cells, T-helper cells, or T-cytotoxic cells. Analysis of the model on a RAG-1 (recombination activating gene-1)deficient background, lacking functional lymphocytes, did not change the observed disease and pathology compared with immunecompetent mice. We conclude that this model provides the opportunity to study the consequences of adult oligodendrocyte death in the absence of primary axonal injury and reactive cells of the adaptive immune system. Our results indicate that if the blood-brain barrier is not disrupted, myelin debris is not removed efficiently, remyelination is impaired, and axonal integrity is compromised, likely as the result of myelin detachment. This model will allow the evaluation of strategies aimed at improving remyelination to foster axon protection.

\section{Introduction}

Altered interactions between myelinating glia and the underlying axon, originating in the glial cell, are often at the heart of diseaseassociated axonal degeneration in the peripheral nervous system and CNS. This observation is prevalent in hereditary motor and sensory neuropathies (Suter and Scherer, 2003), in PelizaeusMerzbacher disease/spastic paraplegia type-2 due to proteolipid

Received Sept. 26, 2010; revised 0ct. 30, 2010; accepted Nov. 5, 2010.

This work was supported by the Swiss National Science Foundation and the National Center for Competence in Research, Neural Plasticity and Repair. We thank Mike Peacock for his valuable help with electron microscopy protocols, Dr. Frank Heppner for help and advice on pathology issues, Dr. Urs Odermatt for providing antibodies, Dr. Frank Costantini for transgenic animals, Dr. Michael Wegner for valuable advice, the Light Microscopy Centre and the Electron Microscopy Centre of the Eidgenössische Technische Hochschule Zürich for excellent support, and the members of the Suter laboratory for many fruitful discussions.

Correspondence should be addressed to Dr. Ueli Suter, Institute of Cell Biology, ETH Hönggerberg, HPM E39, Schafmattstrasse 18, CH-8093 Zürich, Switzerland. E-mail: usuter@cell.biol.ethz.ch.

T. Mueggler's present address: F. Hoffmann-La Roche Ltd., Pharmaceutical Research Neuroscience, CH4070 Basel, Switzerland.

L. C. Bachmann's present address: Brain Research Institute, Department of Neuromorphology, University of Zürich and ETH Zürich, CH-8057 Zürich, Switzerland.

DOI:10.1523/JNEUROSCI.5035-10.2011

Copyright $\odot 2011$ the authors $\quad 0270-6474 / 11 / 311069-12 \$ 15.00 / 0$ protein 1 ( $P L P 1)$ mutations, and mouse models thereof (Nave and Trapp, 2008; Nave, 2010). Immunological components may accentuate the disease phenotypes (Martini and Toyka, 2004; Kroner et al., 2010). A particular example in this regard is multiple sclerosis (MS) (Compston and Coles, 2008). Historically, the emphasis of research in this disease has been on its inflammatory aspects. Today, the opinion predominates that primary demyelination due to autoimmune mechanisms is followed by secondary axonal degeneration. The latter is the cause of irreversible neurological disability, although it has been questioned recently whether inflammatory demyelination is primary or secondary to neurodegeneration (Trapp and Nave, 2008). MS-associated demyelination may also be caused by different mechanisms in different patients and variable sclerotic plaques, including T-cell- or antibody-mediated demyelination, or primary oligodendrocyte (OLG) cell death (Lucchinetti et al., 2000; Barnett and Prineas, 2004; Barnett and Sutton, 2006).

A genetic mouse model in which intrinsic adult OLG death could be induced in a highly controlled and specific fashion may have major benefits. First, such mice could serve as a valuable animal model of demyelination, in which primary OLG loss oc- 
curs without concurrent effects on axons that are a likely feature of toxin-based models of demyelination. Second, the model may allow studies on the dynamics of myelin clearance and microglia activation after defined OLG death, both major determinants of remyelination (Kotter et al., 2006; Neumann et al., 2009). This is considered to be a critical issue for axon protection (Franklin and Ffrench-Constant, 2008). Third, the potential role of the immune system in the developing disease and pathology can be examined. Fourth, the well developed mouse genetics provide ample opportunities to study the function of different regulatory proteins in the process ensuing induced adult OLG death by appropriate cross-breeding. Fifth, the model is likely to provide novel opportunities to study the mechanistic basis and signaling that is involved in axon-glia interactions, including axonal degeneration secondary to OLG death, in health and disease.

To achieve our goal, we used a tamoxifen (TAM)-dependent PLPCreERT2 allele (Leone et al., 2003) in combination with an improved allele carrying a Cre-dependent diphtheria toxin fragment A (DT-A) transgene in the ubiquitously expressed ROSA26 locus (Brockschnieder et al., 2006). Double-transgenic animals treated with TAM turned out to have many of the desired features and are analyzed here.

\section{Materials and Methods}

\section{Generation, maintenance, treatment, and scoring of mice}

The previously characterized mouse lines PLP-CreERT2 (Leone et al., 2003) and R26:LacZ/DT-A (Brockschnieder et al., 2006) were crossed to obtain a model allowing spatially and temporally controlled induction of OLG cell death. The mouse lines PLP-CreERT2 (Leone et al., 2003) and Cre-dependent R26R-eYFP (Srinivas et al., 2001) were crossed to analyze recombination efficiency. Mice were housed under a normal light/dark cycle (12 h) with standard rodent chow and tap water ad libitum. Genotypes were determined by PCR on genomic DNA derived from ear punch biopsies according to published protocols (Leone et al., 2003; Brockschnieder et al., 2006). Mice at the age of $8-12$ weeks were used. Induction of DT-A or yellow fluorescent protein (YFP) expression was achieved by intraperitoneal injections of $2 \mathrm{mg}$ of TAM (Sigma-Aldrich), dissolved in a sunflower oil/ethanol mixture (10:1), for 5 consecutive days (first injection $=$ day 1 ). Mice were monitored regularly, and their impairments assessed with a specially developed clinical scoring procedure, broadly modeled after scorings used in experimental autoimmune encephalomyelitis (EAE) (Matthaei et al., 1989) (score 0, no observable symptoms; score 1, tremor and ataxia, broadened gait, and reduced grip strength; score 2, severe hindlimb weakness plus pronounced ataxia and tremor; and score 3 , animals temporarily fail to maintain upright position, and both front and hind limbs showed severe weakness). Transition states were scored with half steps. Investigated time points were defined as follows: onset, day 20 referring to first observable impairments (score $\leq 0.5$ ); intermediate, day 34 referring to moderate impairments (score $\sim 1.5$ ); end stage, days $39-42$ when mice reached the strongest impairments (score 3). To obtain a more quantitative measure of behavioral abnormalities, mice were placed on a rotarod apparatus (TSE Systems), and the time spent on the rotating rod while accelerating from 4 to 40 $\mathrm{rpm}$ was measured. Three trials per day in $4 \mathrm{~h}$ intervals were performed every 3-4 $\mathrm{d}$ throughout the disease time course. EAE was induced in C57BL/6j mice by immunization against MOG35-55 peptide according to standard protocols (Mendel et al., 1995). Proliferating cells were labeled by injections of iododeoxyuridine (IdU) ( $80 \mu \mathrm{g} / \mathrm{g}$ body weight) three times daily for 5 consecutive days followed by a $5 \mathrm{~d}$ washout period before killing. All experiments were performed in strict adherence to the Swiss Law for Animal Protection, approved by the veterinary office of the Canton, Zürich, Switzerland.

\section{Magnetic resonance imaging}

During the time course of disease development, animals were analyzed with regard to changes in the T2 relaxation time. For quantitative T2 (qT2), which started at day 3 following induction of gene expression, mice were anesthetized using $1.8 \%$ isoflurane (Abbott) in an oxygen/air $(20 \% / 80 \%)$ mixture applied via a facemask with built-in teeth-bar and placed on a water-heated cradle. Body temperature was monitored using a rectal probe coupled to a fluor optic module (QUASYS AG). Magnetic resonance imaging (MRI) acquisition was performed using a Pharmascan 4.7/16 system (Bruker BioSpin) equipped with a birdcage transmit-receive coil for assessment of qT2. T2 values were obtained using a multi-spin echo sequence with the following parameters: fieldof-view $($ FOV $)=19 \times 19 \mathrm{~mm}$; matrix dimension $=132 \times 132$; in-plane resolution, $144 \mu \mathrm{m}^{2}$; repetition time $=2000 \mathrm{~ms}$; echo time $=10 \mathrm{~ms}$; echo spacing $=10.0 \mathrm{~ms}$; no. of echoes, 14 ; no. of averages $=6$; and slice thickness $=0.8 \mathrm{~mm}$. T2 maps were calculated using Biomap (M. Rausch, Novartis). Maps from the same animal were coregistered, and T2 values were plotted as a function of time for the defined regions of interest (ROIs) [frontal cortex (fx), cerebellar white matter (cb), brainstem (bs), anterior commissure (ac)] (Fig. 1D). All data are given as mean \pm SEM. Data were statistically analyzed using repetitive ANOVA $(n=2$ control and 4 experimental animals).

\section{Tissue preparation}

Mice were deeply anesthetized using $150 \mathrm{mg} / \mathrm{kg}$ pentobarbital (Esconarkon, Streuli Pharma AG) and transcardially perfused with heparin (250 $\mathrm{mg} / \mathrm{L}$, Sigma-Aldrich) in PBS followed by fixation with $4 \%$ paraformaldehyde/PBS (Sigma-Aldrich) for light microscopy or with 3\% glutaraldehyde and $4 \%$ paraformaldehyde in $0.1 \mathrm{~m}$ phosphate buffer, $\mathrm{pH} 7.4$, for electron microscopy. Brain and spinal cord tissues were dissected and postfixed overnight at $4^{\circ} \mathrm{C}$. For paraffin sectioning, tissues were dehydrated, embedded in paraffin (Medite), sectioned into $5 \mu \mathrm{m}$ coronal or sagittal sections using a rotary microtome (HM355S, Microm), and taken up on polysine-coated glass slides (Menzel). For cryosectioning, tissues were cryoprotected with $30 \%$ sucrose $/ \mathrm{PBS}$ at $4^{\circ} \mathrm{C}$ overnight, embedded in OCT (Medite), sectioned into $10 \mu \mathrm{m}$ sections using a cryostat (HM560, Microm), and taken up on Superfrost Plus glass slides (Menzel). For semithin and ultrathin resin sectioning, tissues were postfixed and contrasted with $2 \%$ osmium tetroxide (EMS), dehydrated with acetone, embedded in corrected Spurr's resin (EMS) (Ellis, 2006), sectioned into $0.5 \mu \mathrm{m}$ or $80 \mathrm{~nm}$ sections using a fine microtome (Ultracut E, Leica), and taken up on standard glass slides (Menzel) or copper EM grids (EMS). Semithin sections for light microscopy were contrasted with $1 \%$ toluidine blue (Sigma-Aldrich), and ultrathin sections for electron microscopy were contrasted with $3 \%$ uranyl acetate and $1 \%$ lead citrate.

\section{Light microscopy}

Luxol-Fast-Blue (LFB) and Luxol-Nissl (L-N) stainings were executed according to standard procedures. In short, sections were deparaffinized, rehydrated to $95 \%$ alcohol, heated in $0.1 \%$ LFB (Sigma-Aldrich) in a microwave, and differentiated with $0.05 \%$ lithium carbonate (SigmaAldrich) and alcohol. For L-N, counterstaining with cresyl violet acetate was used (Sigma-Aldrich). For Oil Red-O staining, cryosections were postfixed, incubated in 60\% isopropanol, stained in Oil Red-O staining solution [0.3\% Oil Red-O (Sigma-Aldrich) in 60\% isopropanol] for 20 min, and washed in $60 \%$ isopropanol and $\mathrm{H}_{2} \mathrm{O}$. For costainings, Oil Red-O staining was performed after incubation with $\mathrm{ABC}$ reagent mix. Fluoro-Jade C staining was executed according to published protocols (Schmued et al., 2005). For immunohistochemistry on paraffin sections, samples were deparaffinized, rehydrated, and exposed to heat-mediated antigen retrieval using a tissue microwave oven (Medite). Endogenous peroxidase was blocked with $3 \% \mathrm{H}_{2} \mathrm{O}_{2}$ /methanol (Sigma-Aldrich), followed by blocking and permeabilization with blocking buffer $[10 \%$ fetal calf serum (Brunschwig), 1\% bovine serum albumin, and $0.1 \%$ Triton X-100 (both Sigma-Aldrich) in PBS], and incubation with primary antibodies [rat-anti-myelin basic protein (MBP), 1:300; AbD, Serotec), rabbit-anti-degMBP (1:1000; Millipore), rabbit-anti-neurofilament $\mathrm{M}$ (NF-M) (1:300; Millipore), rabbit-anti-Iba-1 (1:300; WAKO Chemicals), rabbit-anti-GFAP (1:500; Dako), rat-anti-B220 (1:200; BD Biosciences), rat-anti-CD4 (1:200; BD Biosciences), and rat-anti-CD8 (1: 200; BD Biosciences)] overnight at $4^{\circ} \mathrm{C}$ in blocking buffer. Sections were washed, incubated with corresponding biotinylated secondary antibodies (1:500; Jackson ImmunoResearch) followed by incubation with Vec- 
A

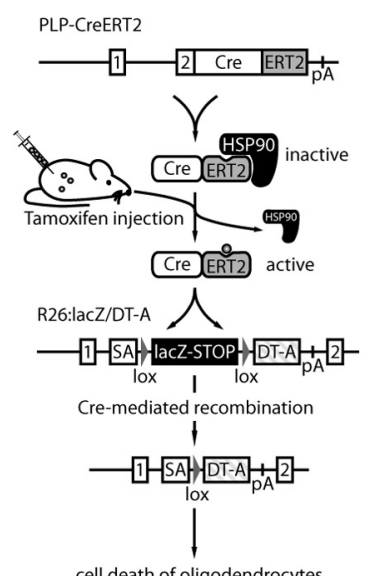

cell death of oligodendrocytes
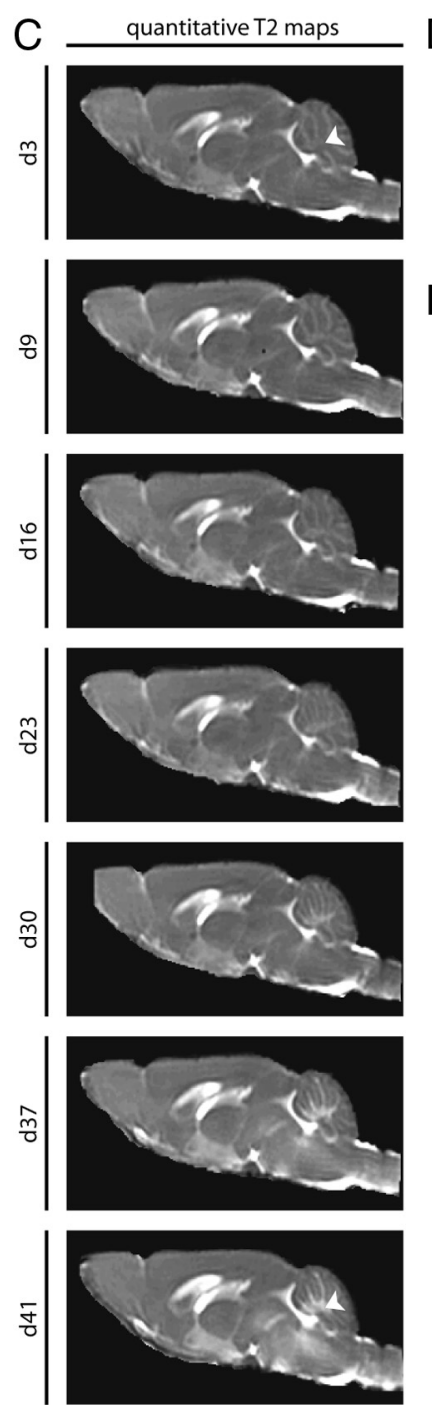
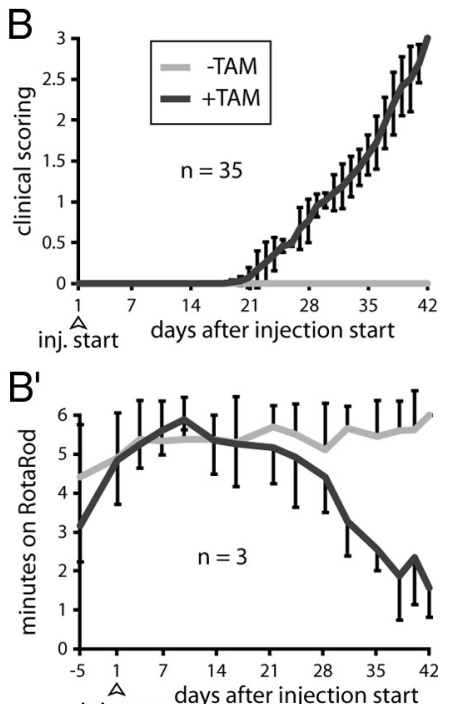

inj. start days after injection start
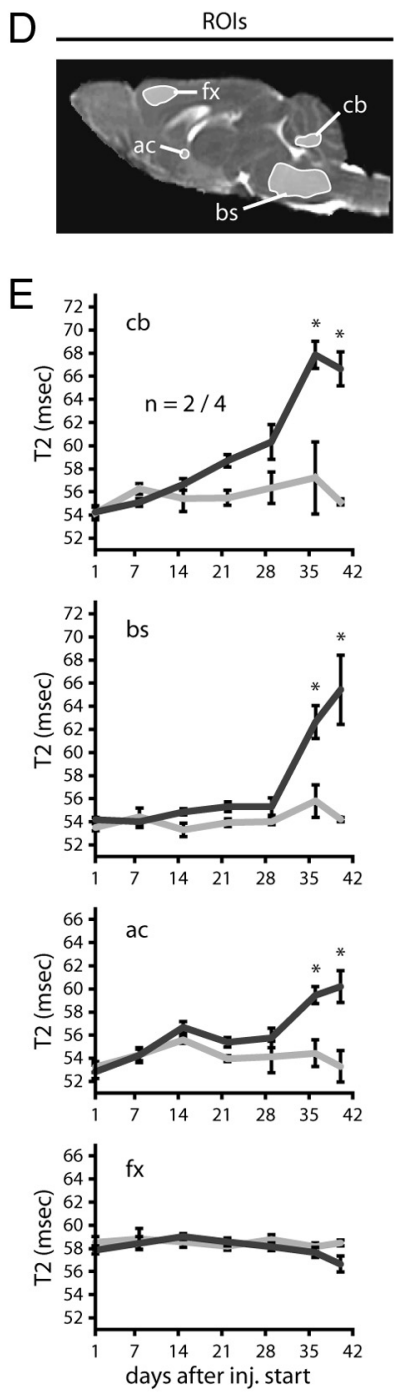

Figure 1. Genetic basis and longitudinal assessment of disease development after induction of adult oligodendrocyte cell death. $\boldsymbol{A}$, Genetic basis for spatially and temporally controlled ablation of adult OLGs. The inducible PLP-CreERT2 line was crossed with the Cre-dependent R26:lacZ/DT-A line to ablate OLGs after TAM injection. $B, \boldsymbol{B}^{\prime}$, Time course of clinical appearance in 2-3-month-old PLP-CreERT2/DT-A double-transgenic mice, with or without TAM injections, as judged by clinical scoring $(\boldsymbol{B})$ and correlative performance in the rotarod test $\left(\boldsymbol{B}^{\prime}\right)$. $\boldsymbol{C}$, tastain $\mathrm{ABC}$ reagent mix (Reactolab), and developed with $\mathrm{DAB}$ metal enhancer solution (Thermo Scientific). Sections were counterstained with cresyl violet acetate and dehydrated.

For immunofluorescence on cryosections, samples were postfixed with $4 \%$ paraformaldehyde followed by blocking and permeabilization with blocking buffer $[10 \%$ fetal calf serum (Brunschwig), $1 \%$ bovine serum albumin, and 0.1\% Triton X-100 (both Sigma-Aldrich) in PBS]. Sections were then incubated with primary antibodies [goat-anti-Sox10 (1:50; Santa Cruz Biotechnology), rabbit-anti-Olig2 (1:500; Millipore), rabbit-anti-NG2 (1: 500; Millipore), mouse-anti-IdU (1:50; BD Biosciences), rabbit-anti-GFP (1:200; Invitrogen), rabbit-anti-NF-M (1:300, Millipore), mouse-antinonphosphorylated neurofilaments (SMI32; Abcam), mouse-anti-amyloid precursor protein (APP) (1:400; Millipore), Fluoro-Jade C (0.0001\%; Millipore), mouse-anti-Caspr (1:400; NeuroMab), and mouse-anti-Kv1.2 (1:200; NeuroMab] overnight at $4^{\circ} \mathrm{C}$ in blocking buffer. For IdU/Olig2 stainings, sections were exposed to antigen retrieval according to IdU supplier recommendations before antibody incubation. Sections were washed, incubated with corresponding fluorescence-coupled secondary antibodies (1: 500; Jackson ImmunoResearch), and counterstained with DAPI (SigmaAldrich). Stained and processed sections were documented using an AxioImager Microscope and an AxioCam MRc5 (Zeiss). Image Processing was performed using Axiocam (Zeiss) and Photoshop (Adobe Systems).

\section{Electron microscopy}

Contrasted ultrathin resin sections were documented using a Morgani 268 transmission electron microscope (FEI). Image Processing was performed using Photoshop (Adobe Systems).

\section{Evans blue permeability assay}

Influx of Evans blue-labeled serum albumin into brain tissue was assessed according to published procedures (Ogunshola et al., 2006). In short, a $1 \%$ solution of Evans blue dye $(2 \mu \mathrm{g} / \mathrm{g})$ was injected intravenously $1 \mathrm{~h}$ before killing. Mice were anesthetized and transcardially perfused with PBS, and brain tissue dissected. Dye retained in the tissue was extracted with formamide, and absorbance was measured at $620 \mathrm{~nm}$.

\section{Quantifications}

Vacuole counting. Tissue vacuoles were blind counted manually on MBPstained paraffin sections on images acquired at $40 \times$ magnification using ImageJ counting software (open source software: http://rsbweb.nih.gov/ ij/), counting eight random FOVs on four different sectioning levels per tissue of interest. Data are given as mean \pm SEM and statistically analyzed using Student's $t$ test ( $n=3$ animals).

Counting of Sox $10^{+}$cells and recombination efficiency. Sox $10^{+}$cells were blind counted manually on Sox10/DAPI-, SOX10/YFP/DAPI-, or NG2/YFP/DAPI-stained sections on images acquired at $10 \times$ magnification using ImageJ, counting four random FOVs on two different sectioning levels per tissue of interest. Data are given as mean \pm SEM and statistically analyzed using Student's $t$ test $(n=3)$.

Counting of proliferating oligodendrocyte precursor cells. Proliferative oligodendrocyte precursor cells (OPCs) were blind counted manually on Olig2/IdU/DAPI-stained sections on images acquired at $40 \times$ magnification using ImageJ, counting 10 random FOVs on at least three different sectioning levels per tissue of interest. Data are given as mean \pm SEM and statistically analyzed using Student's $t$ test $(n=3)$.

Analysis of g-ratios. Corpus callosum g-ratios were determined from $>100$ healthy-appearing myelinated axons per animal on electron micrographs by manually measuring the areas of axon and myelin using Photoshop and calculating g-ratio $=($ axon area/fiber area $)$. Data were

$\leftarrow$

Quantitative MRI T2 maps of a representative animal during the time course of disease development after TAM injections. Note the pronounced hyperintensities in cerebellar core white matter (arrowheads), but also in brainstem and midbrain, first detectable but not yet significant around symptomatic onset (day 23). D, Representative T2 map with superimposed ROIs used for quantifications. $E$, Quantitative T2 values derived from ROls in cb, bs, ac, and fx. Significance in the increase of T2 relaxation times was reached at day 37 in the analyzed white matter (cb, bs, and ac) but not gray matter (fx) regions. Data are given as mean \pm SEM. ${ }^{*} p<0.05$, repetitive ANOVA. 
dot plotted or given as mean \pm SEM and statistically analyzed using Student's $t$ test $(n=3)$.

Analysis of SMI32 immunofluorescence. SMI32 immunofluorescence intensity was measured on images acquired at fixed settings of SMI32stained cryosections of cerebellar core area at $20 \times$ magnification using four FOVs on two different sectioning levels per animal. Images were analyzed using ImageJ. Mean intensity was calculated by dividing mean gray values by the area above a threshold value. Image intensity threshold was set to exclude background signal. Mean of controls was set to 1 , and data are given as mean \pm SEM of relative staining intensity and were statistically analyzed using Student's $t$ test $(n=3)$.

Counting of nodes of Ranvier. Caspr- or Kv1.2-stained spinal cord sections were imaged at $63 \times$ magnification at two different focal planes per FOV, using five random FOVs per animal. The two focal planes were overlaid using Photoshop, and the specific pattern of Caspr ${ }^{+}$paranodes or Kv1.2 $2^{+}$juxtaparanodes were identified and counted manually in a blinded manner. Data are given as mean \pm SEM and statistically analyzed using Student's $t$ test $(n=3)$.

Quantification of microglia. Iba- ${ }^{+}$cells were blind counted manually on MBP-stained paraffin sections on images acquired at $40 \times$ magnification using ImageJ, counting six FOVs on two different sectioning levels. Data are given as mean \pm SEM and statistically analyzed using Student's $t$ test $(n=3)$.

\section{Results}

Characterization of behavioral changes of a genetically controlled mouse model of induced adult oligodendrocyte cell death reveals a highly consistent disease development

Our aim was to establish a mouse model that allowed defined induction of genetically controlled cell death of adult OLGs. To achieve this, we crossed a mouse line carrying an optimized TAM-inducible version of Cre (CreERT2), under PLP gene transcriptional control (Leone et al., 2003), with a mouse line containing a Cre-dependent DT-A expression cassette (Fig. 1A) (Brockschnieder et al., 2006). Double-transgenic offspring were treated with $2 \mathrm{mg} / \mathrm{d}$ TAM for 5 consecutive days at the age of 2-3 months (young adults). These animals developed a highly consistent disease course, regarding onset of clinical signs and progression (Fig. $1 B$ ), as evaluated using a specifically developed scoring assay (see Materials and Methods). Such clinical changes were never observed in control animals (vehicle-injected doubletransgenic animals or TAM-treated single-transgenic animals of either mouse line; data not shown). Clinical progression in TAMtreated double-transgenic animals (henceforth referred to as "experimental mice") was characterized by an initial lag period of 3 weeks without evident disease signs. Around $21 \mathrm{~d}$ after injection start, the experimental mice showed increasing tremor and ataxia, motor deficits accompanied by muscle atrophy, and weight loss. The severity of disability increased steadily over the next 3 weeks, and the animals could not be kept longer on welfare grounds. To obtain a quantitative measure of the increasing deficits, we analyzed experimental and control mice using the rotarod test (Fig. $1 B^{\prime}$ ). The results mirrored our clinical scoring, thus confirming the strikingly uniform and steady time course of disease progression. Thus, our data show that induced cell death of adult OLGs generates a defined and clinically highly reproducible mouse phenotype.

\section{Longitudinal magnetic resonance imaging is a suitable method to follow the consequences of adult oligodendrocyte loss}

Following pathological changes longitudinally in the same living animal has many self-evident experimental advantages. We chose to examine whether this is feasible in our experimental mice by carrying out serial MRI assessing quantitative T2 values through- out disease progression. Parasagittal T2 maps obtained from experimental animals showed consistent alterations over the time course of disease progression as evidenced by increasing hyperintensities in distinct brain areas (Fig. 1C). Qualitative evaluations revealed first effects around disease onset at day 23, especially pronounced in cerebellar core white matter, brainstem regions of pons and medulla, and midbrain (Fig. 1C). Quantifications of T2 relaxation times in defined ROIs (Fig. $1 D$ ) revealed no alterations in control mice across the longitudinal study with average T2 values of $55 \pm 3 \mathrm{~ms}$. T2 values of the experimental group increased over time (i.e., with values for the cerebellar ROI changing from a baseline of $54 \pm 0.5$ to $68 \pm 1 \mathrm{~ms}$ at end stage). This pronounced increase in $\mathrm{T} 2$ matches disease progression in white and mixed matter tissues including cerebellar core, anterior commissure, and brainstem, but was not detectable in gray matter regions like frontal cortex (Fig. $1 E$ ). Dilation of ventricles was not an obvious contributing feature, although we cannot exclude occasional minor effects. We did not observe significant changes of T2 values in the various brain regions of control mice throughout the observation period. We conclude that longitudinal MRI analysis is suitable to follow impairments due to induced loss of OLGs in time and space in accordance with the observed disease development time course. Thus, this method can potentially be used to examine the benefits of experimental treatment strategies aimed at replacing the lost OLGs either by activating resident stem/precursor cells or by transplantation.

\section{Acute loss of adult oligodendrocytes leads to myelin vacuolation}

To assess changes in tissue morphology in our experimental mice and to correlate these to the behavioral and imaging data, we performed immunostaining at representative time points of phenotypic development: before TAM-mediated induction; at onset of clinical signs at day 20, intermediate stage at day 34 , and end stage at days 39-42 (Fig. 2). First, we examined myelin by immunolabeling for MBP. Myelin staining was progressively reduced over time in the cerebellar white matter, brainstem, midbrain, and spinal cord white and gray matter (Fig. $2 \mathrm{~A}$ ). We also observed a widespread progressive vacuolation (status spongiosus) in white matter that was present at the onset of clinical signs and increased in size and number over time (Figs. $2 A^{\prime}, 3 A, B$ ). Next, we examined neurons using NF staining, which revealed a progressive loss of staining intensity. These findings are consistent with secondary axonal damage due to primary loss of adult OLGs and the associated myelin alterations (Fig. 2B).

The severity of vacuolation differed throughout the neuroaxis. Most severely affected were the cerebellar white matter, brainstem, and spinal cord white matter, while the spinal cord gray matter showed no prominent vacuole load (Fig. 3A). Analysis of vacuoles in the severely affected regions of cerebellar white matter and brainstem showed a steady increase over time, in parallel to the progression of clinical signs (Fig. $3 B$ ). Interestingly, myelinated regions of the forebrain were variably affected, being especially severe in the anterior commissure while the corpus callosum was only mildly altered at end stage (Fig. $3 A$ ).

To determine the cellular origins of the status spongiosus (Adornato and Lampert, 1971), we analyzed first toluidine bluestained resin sections. As shown in the representative region of the spinal trigeminal tract of experimental mice (Fig. $3 C$ ), the observed tissue abnormalities were similar in distribution to those revealed by myelin staining (Fig. 2A). Vacuoles were restricted to single myelin lamellae at initial stages, but over time the expanding vacuoles caused widespread tissue disruption. Ul- 
A MBP / Niss

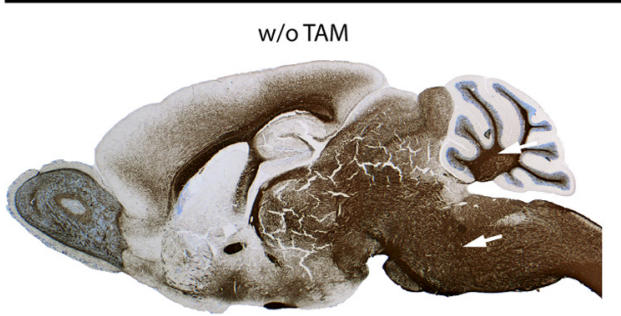

intermediate

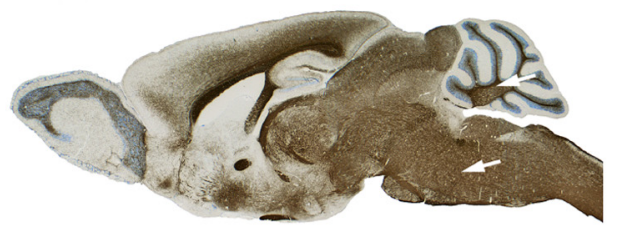

$A^{\prime}$
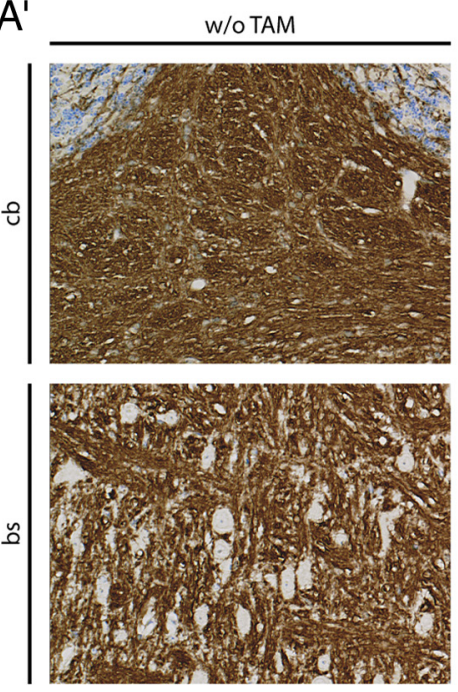
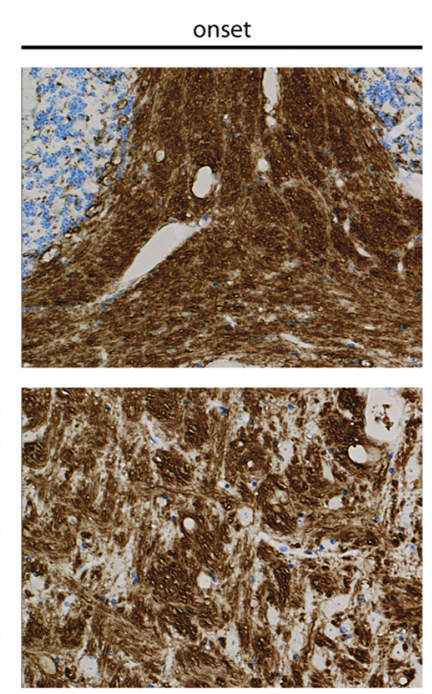

onset

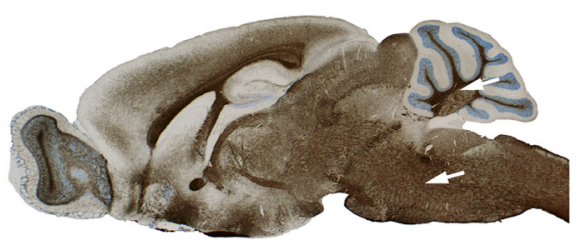

end stage
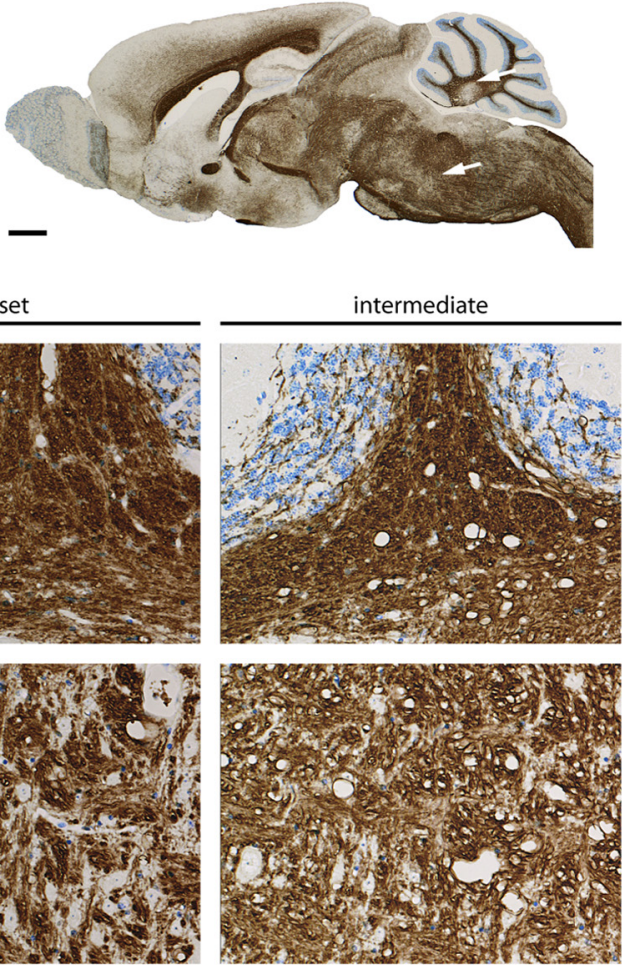

w/o TAM

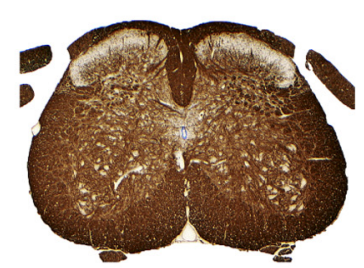

end stage

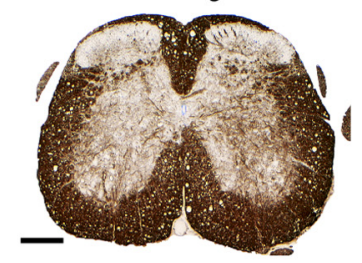

end stage
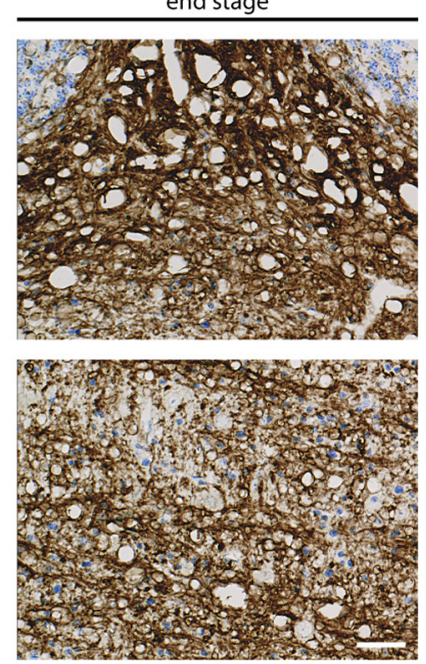

B

NF / Nissl

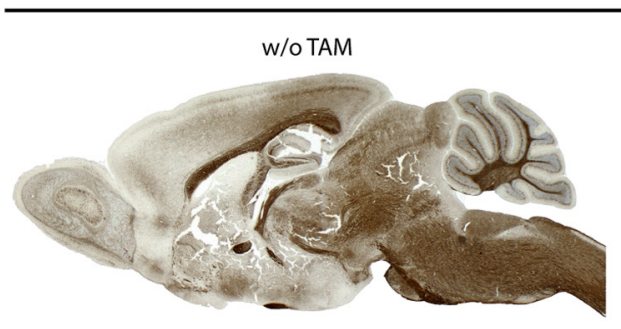

intermediate

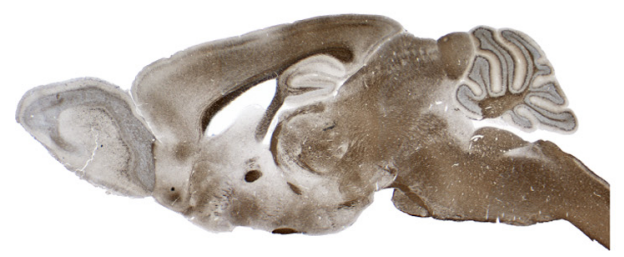

onset

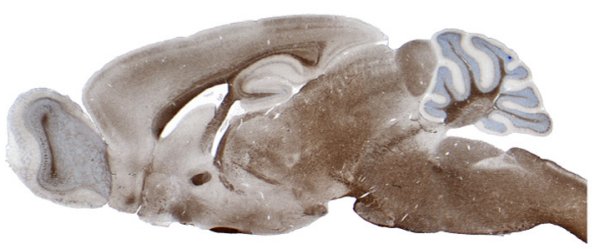

end stage

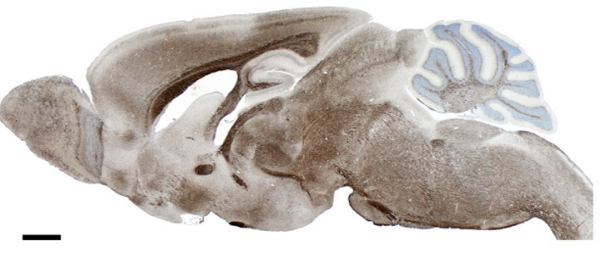

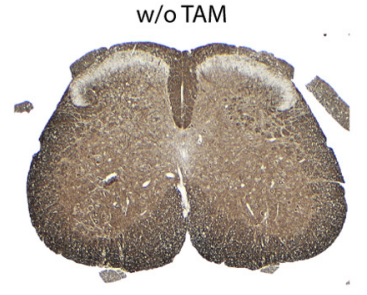

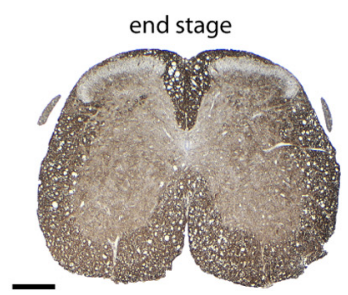

Figure 2. Histological assessment of brain and spinal cord tissue after induction of adult oligodendrocyte loss. $A$, MBP staining on brain sagittal sections (left) and spinal cord cross sections (right) at different time points of disease progression. Note the reduced MBP staining over time, most pronounced in cerebellar core white matter and brainstem (arrows). $\boldsymbol{A}^{\prime}$, Magnifications of $c b$ and bs regions show reductions of myelin staining over time and remarkably increasing tissue vacuolation. $\boldsymbol{B}$, NF staining on brain sagittal sections (left) and spinal cord cross sections (right) at different time points of disease progression. Note the reduced NF staining intensity in impaired regions, especially at end stage. Scale bars: $\boldsymbol{A}, \boldsymbol{B}$, brain overviews, $1 \mathrm{~mm} ; \boldsymbol{A}, \boldsymbol{B}$, spinal cord sections, $200 \mu \mathrm{m} ; \boldsymbol{A}^{\prime}, 20 \mu \mathrm{m}$. 

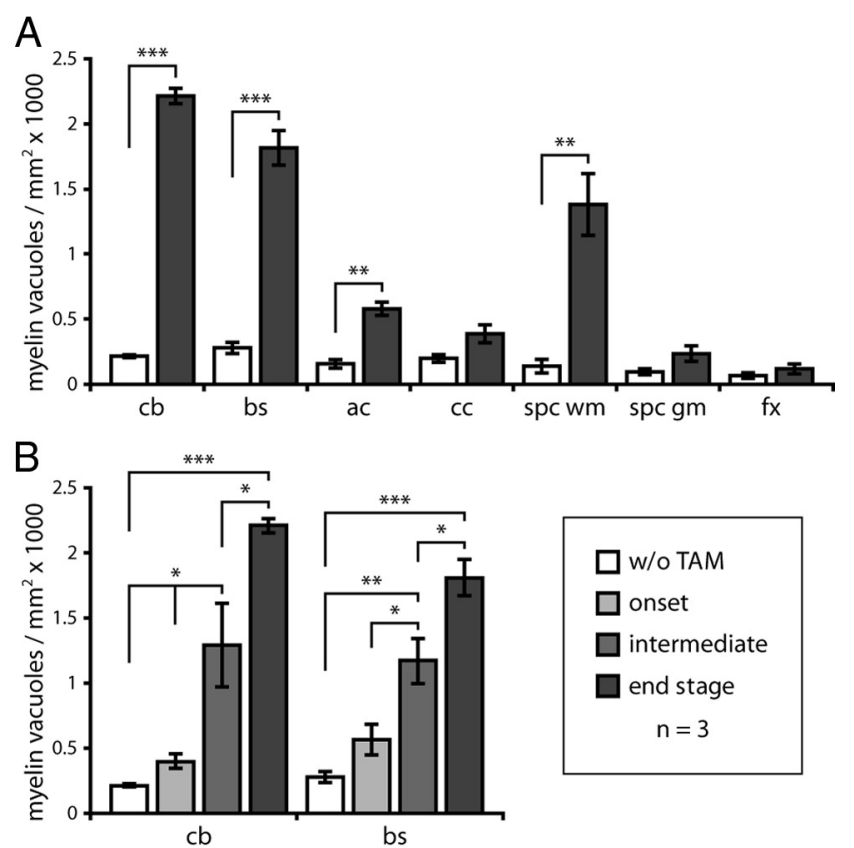

C
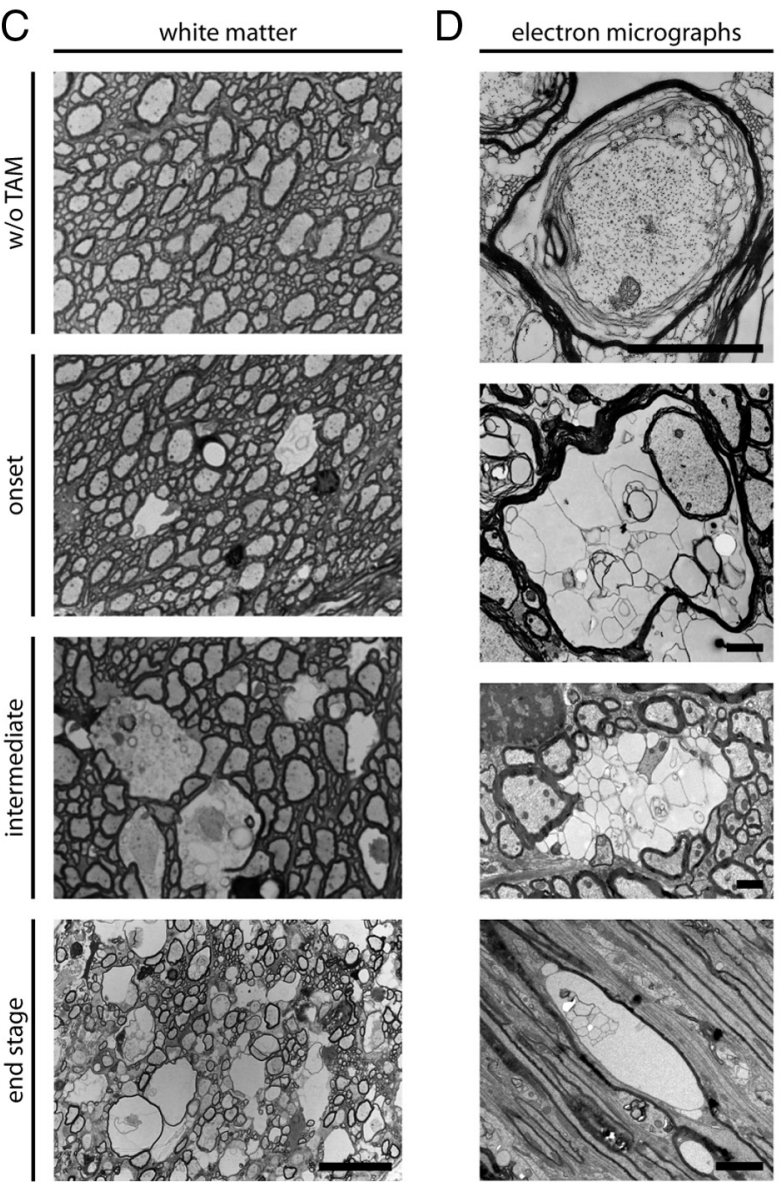

Figure 3. Tissue vacuolation after induction of adult oligodendrocyte death. $A$, Tissue vacuolation at end stage of mice affected by genetically induced $0 L G$ cell death: $c b$, bs, ac, corpus callosum (cc), spinal cord white (spc wm), and gray (spc gm) matter, and fx. B, Accumulation of vacuoles is most prominent in severely affected white matter tissues, and vacuoles appear and increase in numbers during disease development as quantified by manual counting on histological sections. C, Morphology of representative spinal trigeminal tract regions of the brainstem on toluidine blue-stained resin sections. Vacuoles appear in parallel to clinical signs and increase in both size and numbers while impairment of the mice progresses. Widespread tissue vacuolation is observed at end stage. Note the abundance of disrupted, degenerative, but not trastructural analysis confirmed that vacuolation was due to alteration of myelin membranes (Fig. 3D). Vacuoles arose due to splitting at various places within the myelin sheath, between the axolemma and the myelin sheath, or by a combination of both. Membrane splitting and vacuolation occurred locally along the internode, with the rest of the myelin sheath remaining compact (Fig. 3D). In summary, experimental mice show major progressive myelin vacuolation in parallel with the development of clinical signs.

\section{Efficient elimination of oligodendrocytes by genetically induced cell death triggers oligodendrocyte precursor proliferation}

To determine the efficacy of induction of OLG cell death in our experimental animals, we used the pan-oligodendrocyte lineage marker Sox10 (Wegner, 2001; Rivers et al., 2008). OLG densities varied markedly between different CNS regions in control animals (supplemental Table 1, available at www.jneurosci.org as supplemental material). Quantification revealed a decrease of Sox $10^{+}$cells in affected areas in experimental animals at end stage (reductions ranged between 60 and 80\%) (Fig. 4A). We selected cerebellar white matter (reduction of $78 \pm 5.3 \%$ ), brainstem (reduction of $55 \pm 6.7 \%$ ), and corpus callosum (reduction of $65 \pm 2.8 \%$ ) for further analysis due to their differences in Sox $10^{+}$cell numbers and morphology at end stage. We found a significant reduction of Sox $10^{+}$cells already $3 \mathrm{~d}$ after the start of TAM injections, with progression at $6 \mathrm{~d}$, and lowest observed levels reached at $11 \mathrm{~d}(\sim 80 \%$ reduction in all three regions examined) (Fig. $4 B$ ). These results are in line with the recombination efficiencies found in the same areas using the Cre-dependent R26:eYFP reporter mouse line in combination with the PLPCreERT2 transgene and identical TAM regimens as in experimental mice (Fig. $4 E$ ). In the same experimental setting, we found $\sim 30 \%$ of $\mathrm{NG}^{+}$OPCs to be recombined, indicating that this population is also affected (Fig. $4 F$ ). We do not know whether this ubiquitous minor loss of OPCs contributes to the resulting phenotype. After disease onset, changes in Sox $10^{+}$cell numbers varied between different regions in experimental mice. The severely affected cerebellar white matter showed no significant increase in Sox $10^{+}$cell density, while in the similarly affected brainstem, Sox $10^{+}$cells increased significantly between intermediate and end stage. The weakly affected corpus callosum showed a significant increase between onset and intermediate stage. Next, we asked whether the observed increases in Sox $10^{+}$ cells might be due to increased OPC proliferation. Thus, we labeled cells with IdU injections, starting 10 days before killing at end stage, and quantified IdU-Olig2 double-positive OPCs. Proliferating Olig ${ }^{+}$cells were highly increased in most affected areas (cerebellar white matter, brainstem, and anterior commissure) but not in the mildly affected corpus callosum or in the strongly affected spinal cord white matter (Fig. 4C,D). Thus, the genetic cell ablation is synchronous and consistent between the different areas that we examined, but subsequent pathology development, including induction of OPC proliferation, varies to some degree.

$\leftarrow$

yet cleared myelin. $\boldsymbol{D}$, Electron micrographs of vacuolated tissue reveal pronounced status spongiosus of myelin sheaths appearing adaxonal, within myelin layers, or even affecting the entire myelin sheath. Vacuolation can also be a local event along an internode. Data are given as mean \pm SEM. ${ }^{*} p<0.05,{ }^{* *} p<0.01,{ }^{* * *} p<0.001$, Student's $t$ test. Scale bars: $\boldsymbol{C}, 20 \mu \mathrm{m} ; \boldsymbol{D}$, $2 \mu \mathrm{m}$. 
A
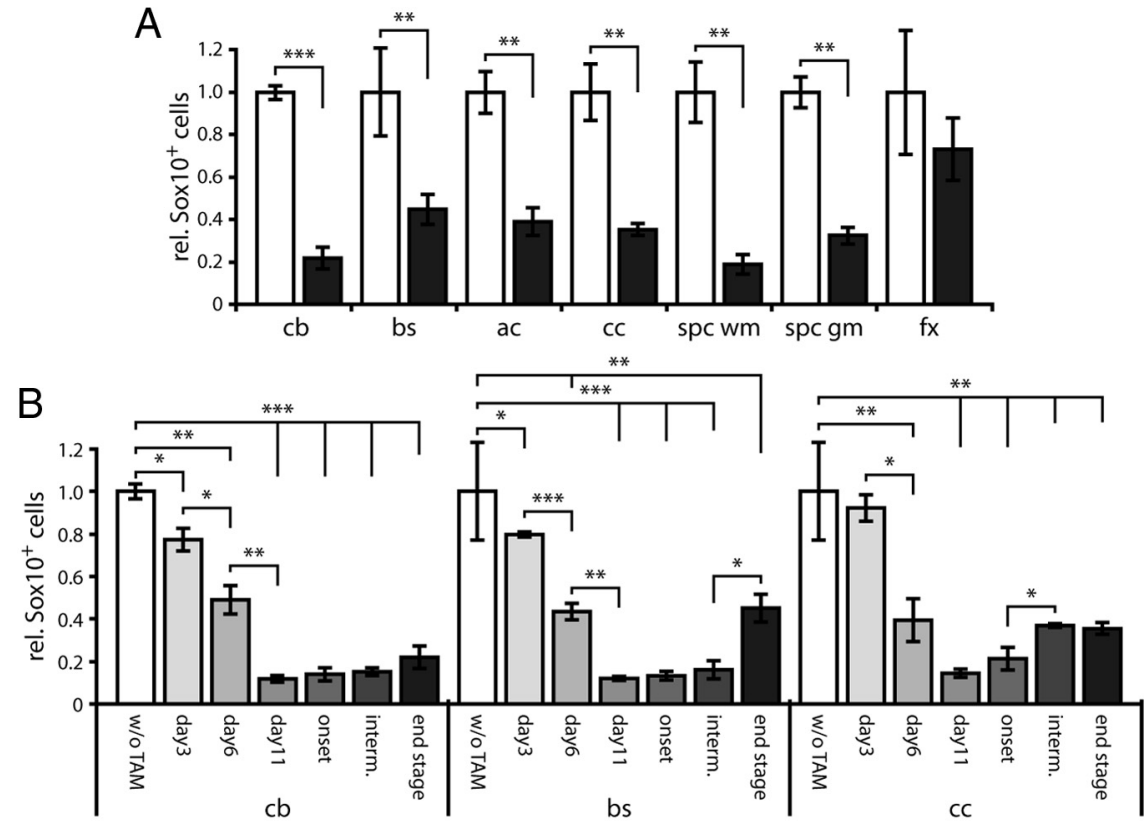

C

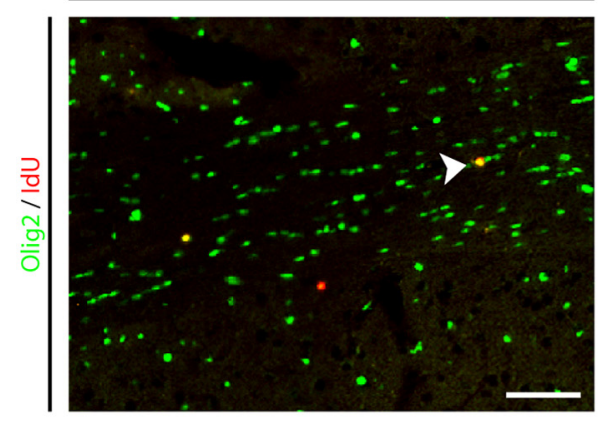

w/o TAM

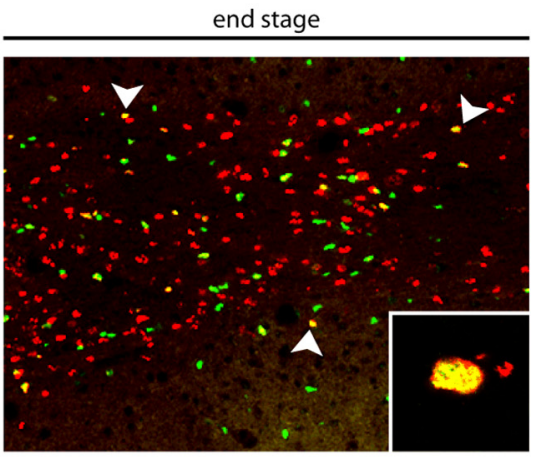

D

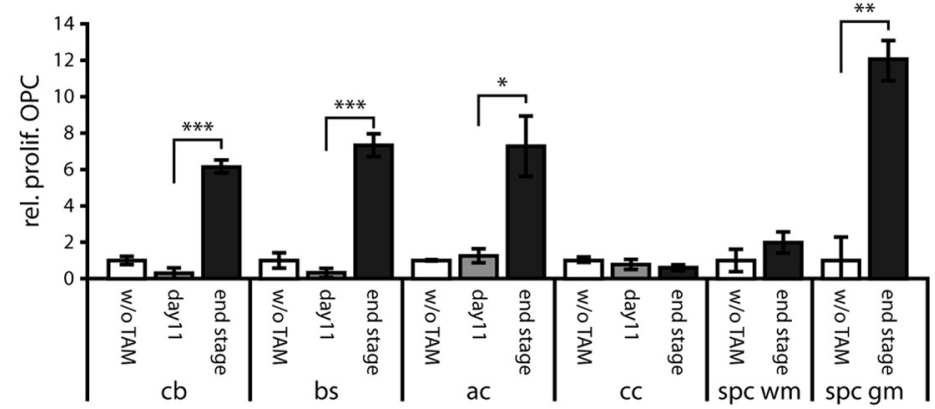

E
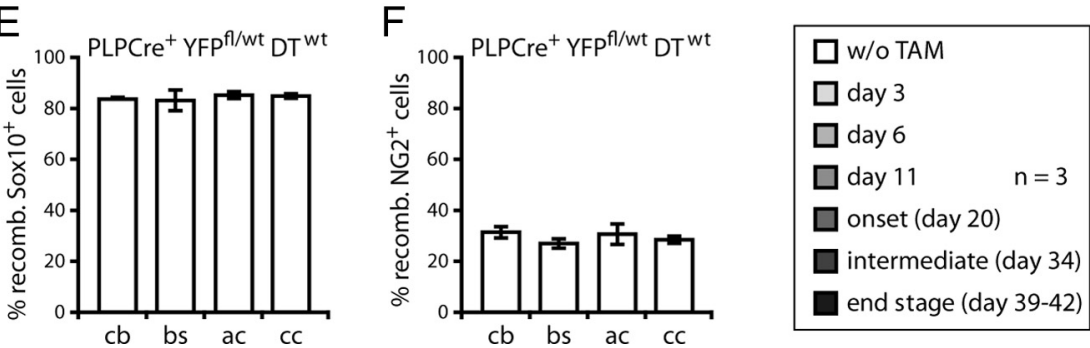

Figure 4. Loss of oligodendroglia and oligodendrocyte precursor proliferation. $\boldsymbol{A}$, Loss of Sox $10^{+}$oligodendrocytic cells following TAM-mediated recombination and subsequent cell-intrinsic expression of DT-A in various CNS areas: $c b, b s, a c$, corpus callosum (cc), spinal cord white matter (spc wm), spinal cord gray matter (spc gm), and fx at end stage of clinical development compared with control mice. Sox $10^{+}$cell numbers are strongly diminished with the exception of the fx. B, Reduction of oligodendroglial cells starts immediately after TAM-induced recombination and reaches the lowest point at day 11 . Numbers remain low with some increases in bs and cc at late time points. C, Proliferating 0lig2 ${ }^{+} \mathrm{OPC}$ in the anterior commissure after labeling for 5 consecutive days with a thymidine analog, starting $10 \mathrm{~d}$ before killing at end stage. Arrowheads mark proliferating OPCs. Inset,
Slow removal of myelin debris in affected areas and impaired remyelination Myelin changes and proliferation of OPCs are often associated with the regenerative process of remyelination. We therefore undertook a detailed electron microscopic analysis of affected tissue since this provides the most reliable means of detecting the thin myelin sheaths typical of remyelination. Even at end stage, large quantities of partially disrupted myelin debris were present (Fig. 3C,D). These findings were corroborated with immunostains revealing widespread degenerated myelin, using an antibody that detects specifically an MBP epitope unmasked during myelin degeneration (Takahashi et al., 2007) (supplemental Fig. $3 C$, available at www.jneurosci.org as supplemental material). In all affected white matter areas, we frequently found phagocytic cells involved in myelin debris clearance, as indicated by myelin debris-filled vacuoles within their cytoplasm (Fig. $5 A$ ), a finding further confirmed as widespread by Oil Red-O/Iba-1 double stainings (supplemental Fig. $3 A, B$, available at www.jneurosci.org as supplemental material). Furthermore, denuded axons were observed (Fig. 5B), although the majority of axons were still associated with disrupted myelin (Fig. 5D) or, less often, normal-appearing myelin. Occasionally, we found abnormally thin but normalappearing myelin sheaths indicative of remyelination (Fig. 5C). However, such remyelinated fibers were sparse and dispersed. Loss of myelin sometimes affected only individual internodes along a given axon, as indicated by the presence of occasional heminodes (Fig. 5E). In the corpus callosum, quantitative analysis of g-ratios revealed no significant difference in experimental mice compared with controls $(0.71 \pm 0.005$ vs $0.70 \pm 0.004)$, although g-ratios $>0.8$ were only found in experimental mice $(10.3 \% \pm 2.62$ vs $2.04 \% \pm 1.09$ in control, $p=0.044$ ) (Fig.

\section{$\leftarrow$}

Single optic confocal microscopy section of exemplary doublepositive cell. D, Quantification of proliferative OPCs shows an increase in all areas analyzed (cb, bs, ac, and spc gm), as well as in cc and spc wm, at end stage but not at day 11 after start of TAM treatment. $\boldsymbol{E}$, Recombination efficiencies are comparable between different CNS regions when analyzed with a PLPCreERT2/Cre-dependent R26:eYFP reporter mouse and the routine TAM injection protocol. $\boldsymbol{F}$, Using the same experimental setup as in $\boldsymbol{E}$, recombination is observed in a fraction of $O P C s$, comparable between the CNS regions analyzed. Data are given as mean \pm SEM. ${ }^{*} p<0.05,{ }^{* *} p<0.01,{ }^{* * *} p<0.001$, Student's $t$ test. Scale bar, $50 \mu \mathrm{m}$. 

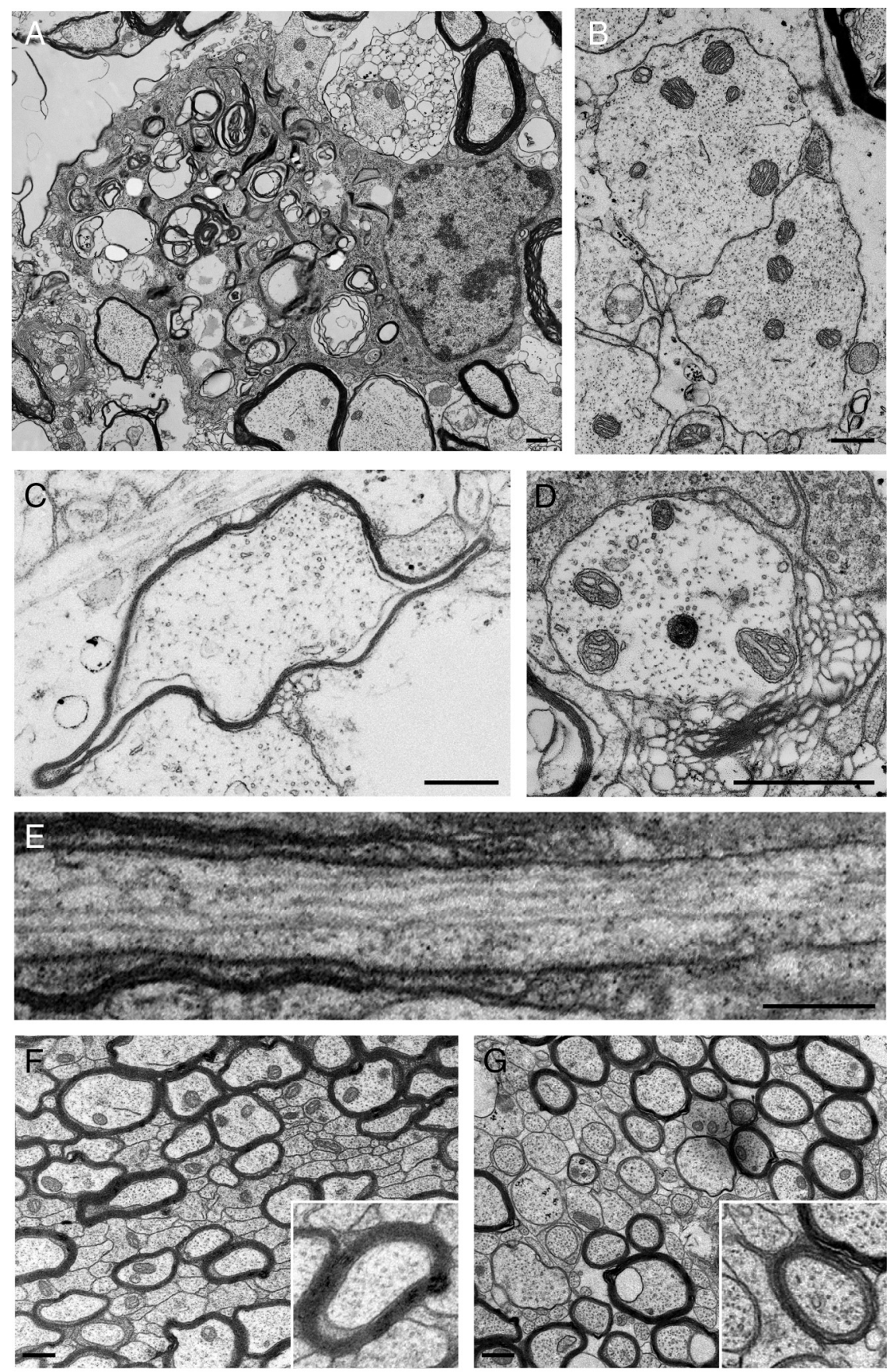

$\mathrm{H}$

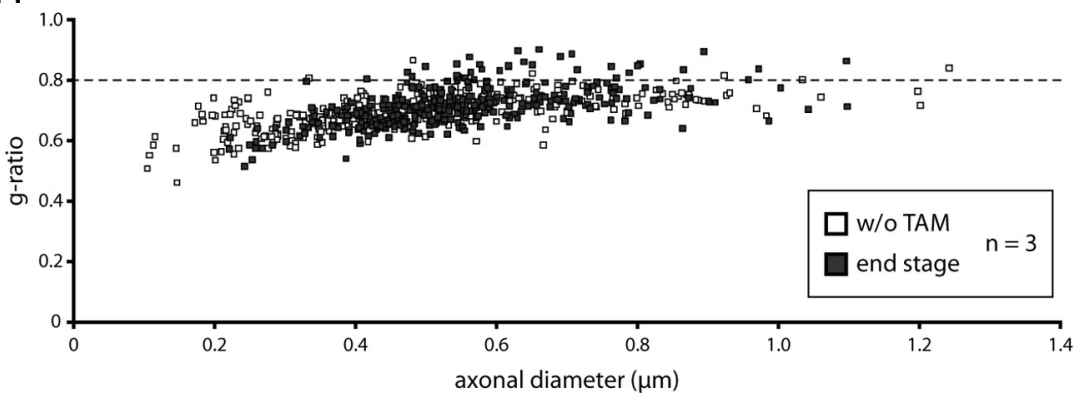

Figure 5. Myelin debris clearance and remyelination. $\boldsymbol{A}$, Phagocytic cells engaged in clearance of myelin debris, as demonstrated by myelin debris-filled vacuoles, were frequently found in damaged tissue at end stage. $\boldsymbol{B}$. Completely denuded axons can be found in strongly affected tissue. $\boldsymbol{C}$, Occasionally, thin myelin sheaths indicative of remyelination were present. $\boldsymbol{D}$, Presence of myelin debris in the vicinity of naked axons, likely to inhibit remyelination. $\boldsymbol{E}$, Demyelination affecting only a selected internode leading to heminode formation. $\boldsymbol{F}, \mathbf{G}$, Comparison of the weakly affected corpus callosum between control $(\boldsymbol{F})$ and experimental $(\boldsymbol{G})$
$5 H)$. Axonal diameters of myelinated axons did not differ between the two groups (data not shown). These data indicate that remyelination probably occurred in the corpus callosum after acute OLG death, although its extent was difficult to assess given that g-ratios of myelinated and remyelinated axons are very similar due to the small diameters of the majority of axons in this tract (Stidworthy et al., 2003). Overall remyelination in our model was not sufficient to cause functional recovery in the experimental mice before the experiment had to be terminated (Duncan et al., 2009).

Genetically targeted cell death of adult oligodendrocytes causes axonal damage Next, we investigated whether the loss of adult OLGs affected axonal integrity. We first performed immunohistochemistry using SMI32 antibodies that specifically recognize a nonphosphorylated neurofilament epitope, in combination with an antibody that recognizes neurofilament M. A striking increase in immunoreactivity in the cerebellar white matter of experimental mice was detected at end stage (Fig. 6A). Consistent with these findings, we also observed increased neuronal staining of APP, an indicator of impaired axonal transport (Fig. 6B), and FluoroJade C, a marker of general axonal/neuronal impairment (Schmued et al., 2005), showed increased signal intensity (Fig. $6 \mathrm{~B}$ ). Morphological observations of axonal accumulation of cell organelles (Fig. $6 E$ ) and axon atrophy (Fig. 3C,D) corroborated these findings. Quantitative analysis of SMI32 immunoreactivity over time revealed a tendency of axonal damage starting with onset of clinical signs and reaching significance at end stage (Fig. $6 C)$. To ascertain changes at nodes of Ranvier, we analyzed Caspr (localized in the paranodal axolemma) and Kv1.2 (juxtaparanodal axolemma) by immunohistochemistry on spinal cord (end stage) longitudinal sections. Both marker proteins were lost at approximately half of nodes of Ranvier in experimental mice (reduction: $\mathrm{Caspr}^{+}$, to $49.4 \pm 3.3 \%, p=$ $0.003 ; \mathrm{Kv} 1.2^{+}$internodes, to $47.3 \pm 5.4 \%$,

$\longleftarrow$

mice at end stage showing some thinner myelin sheaths in the latter, indicative of remyelination. Inserts show magnifications of myelinated axons of comparable size. $\boldsymbol{H}$, Scattergram of g-ratios in relation to axonal diameters reveals a population of thinly myelinated axons with g-ratios $>0.8$ specifically in experimental animals. Note the increased number of axons with g-ratios $>0.8$ in experimental mice. Scale bars, $1 \mu \mathrm{m}$. 

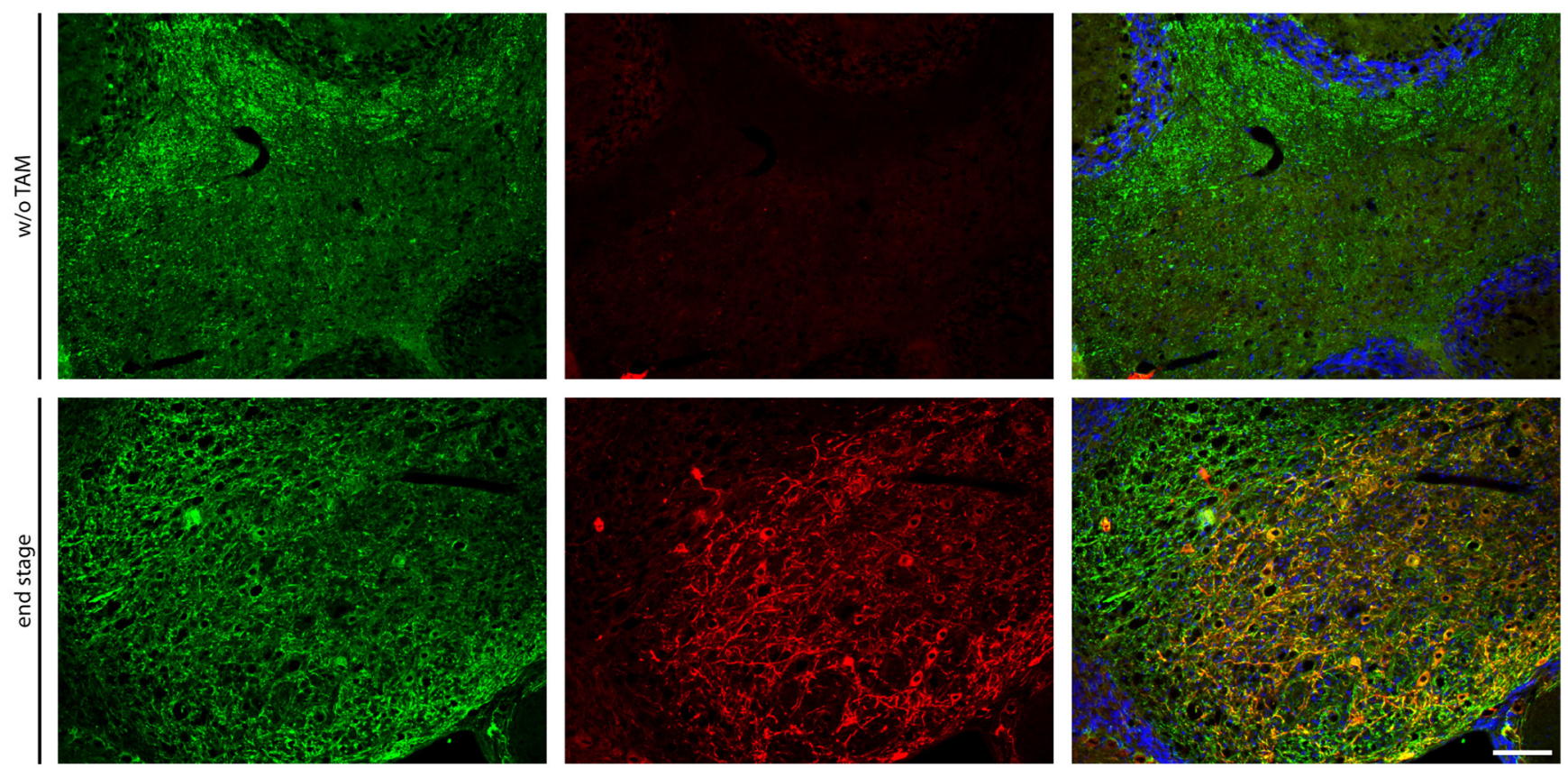

B
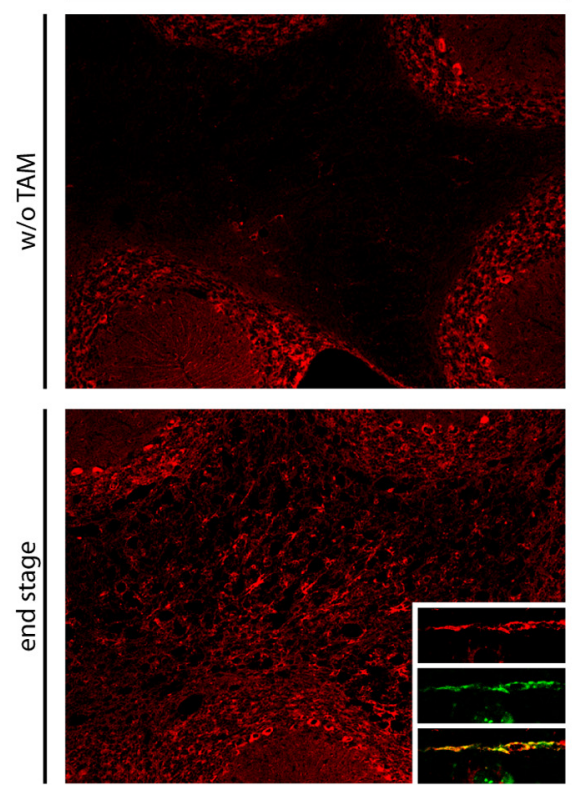

Fluoro-Jade C
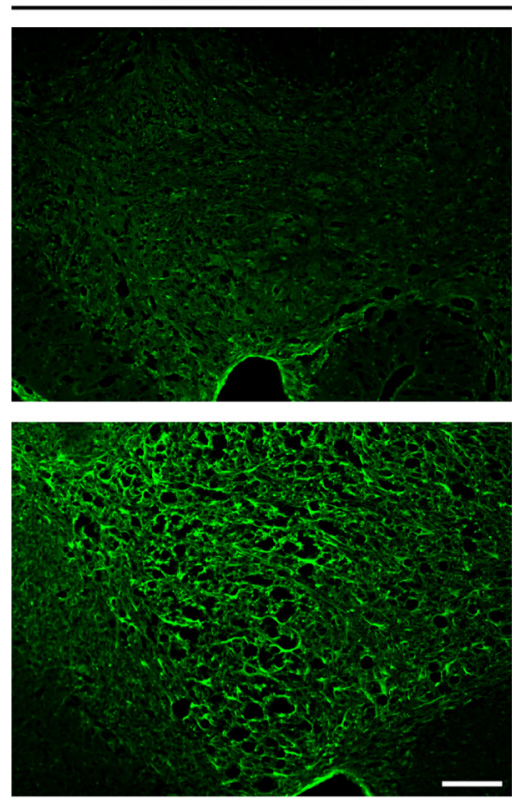

C
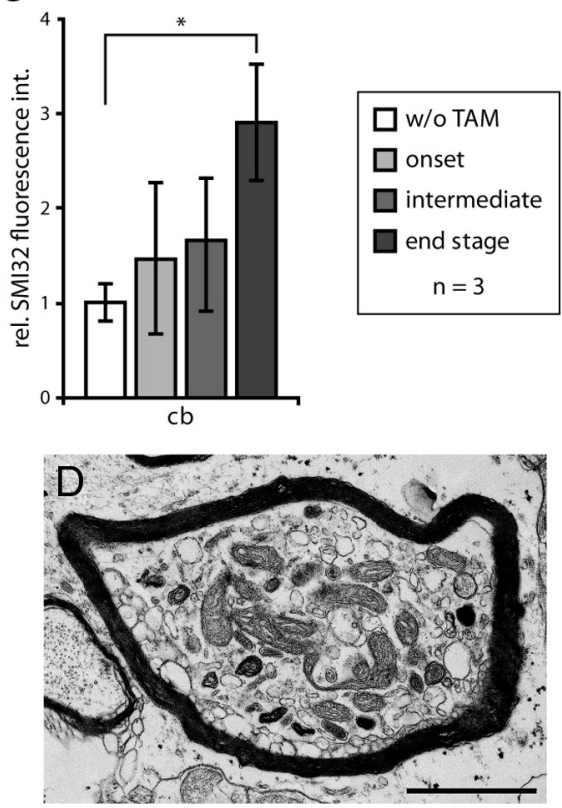

Figure 6. Axonal impairment after induction of adult oligodendrocyte cell death. $A, B$, Animals at end stage show increased immunofluorescent staining for nonphosphorylated neurofilament (SMI32) (A), APP and Fluoro-Jade C (B), indicative of secondary axonal damage in cb. Inset shows single confocal plane optical section of a neuron that was double positive for APP and NF-M. $\boldsymbol{C}$, Quantifications of SMI32 staining over the time course of disease development in cb, reaching significance in increase at end stage. $\boldsymbol{D}$, Exemplary aberrant accumulation of organelles in axon indicative of defective axonal transport. Data are given as mean \pm SEM. ${ }^{*} p<0.05$, Student's $t$ test. Scale bars: $A, B, 100 \mu \mathrm{m} ; \boldsymbol{D}, 2 \mu \mathrm{m}$.

$p=0.002)$. In summary, these data indicate that axons are vulnerable to the death of adult OLGs.

\section{Axonal damage following genetically induced loss of adult} oligodendrocytes occurs independently of the adaptive immune system

It is currently not clear whether axonal loss in a demyelinating disease such as MS, characterized by a maladaptive immune response, is entirely due to inflammation or at least partially to loss of myelin-associated trophic support. To characterize the immune response to white matter damage in our experimental mice, we first used Iba-1 immunohistology. We found widespread activation of cells of the monocyte/macrophage lineage (including microglial cells), especially within affected areas and associated with myelin debris (Fig. 7A; supplemental Figs. $1 A$, $3 B$, available at www.jneurosci.org as supplemental material) accumulating with disease progression (Fig. $7 B$ ). Iba- $1^{+}$cells were also proliferating (supplemental Fig. 2, available at www. jneurosci.org as supplemental material). In parallel, we observed a widespread and pronounced increase of $\mathrm{GFAP}^{+}$astrocytes (supplemental Figs. $1 B, 2$, available at www.jneurosci.org as supplemental material). We next looked for cells of the adaptive 
immune system, but we did not find accumulations of B-cells $\left(\mathrm{B} 220^{+}\right)$, T-helper cells $\left(\mathrm{CD} 4^{+}\right)$, or T-cytotoxic cells $\left(\mathrm{CD}^{+}\right)$in damaged or unaffected regions at end stage (Fig. 7C). These findings suggested an intact blood-brain barrier (BBB) in our experimental mice, which we confirmed by finding no detectable penetration of blood serum albumin into brain tissue when traced with Evans blue dye by immunofluorescence (data not shown) or Evans blue permeability assay at end stage (Fig. 7D). To prove the absence of an adaptive immune component to the phenotype, we crossed the experimental mice onto a recombination activating gene-1 (RAG-1)-deficient background (Mombaerts et al., 1992) that is depleted of functional lymphocytes. When ablation of OLGs was induced in these mice, clinical sign development was indistinguishable from mice with a fully functional immune system (Fig. 7E). There were also no significant differences in tissue vacuolation $\left(2213 \pm 58\right.$ myelin vacuoles $/ \mathrm{mm}^{2}$ in $R A G-1^{w / w}$ vs $2379 \pm 73$ myelin vacuoles/ $\mathrm{mm}^{2}$ in $R A G-1^{0 / 0}$ at end stage, $\left.p=0.1477\right)$, activation of phagocytic cells $(5.55 \pm 0.40$ Iba- $1^{+}$cells $/ \mathrm{mm}^{2}$ in $R A G-1^{w / w}$ vs $5.15 \pm$ 0.58 cells $/ \mathrm{mm}^{2}$ in RAG- $1^{0 / 0}$ at end stage, $p=0.6027)$, or axonal damage $(5.01 \pm 0.55$ relative SMI32 fluorescence intensity in $R A G-1^{w / w}$ vs $5.14 \pm 0.74$ relative SMI32 fluorescence intensity in $R A G-1^{0 / 0}$ at end stage, $p=0.895 ; 1.00 \pm 0.13$ and $0.99 \pm 0.47$ relative SMI32 fluorescence intensity in control animals of the respective genotype). Together, these results suggest that the adaptive immune system is not involved in the pathology following genetically triggered cell death of adult OLGs.

\section{Discussion}

In this article, we show that cell-intrinsic ablation of adult OLGs in the intact CNS provides a highly reproducible model to examine the biology of myelin-axon interactions and tissue repair. Using this model, we provide evidence that efficient removal of myelin benefits from a disrupted BBB: Without this occurring, remyelination is poor and axons are damaged. This axonal injury is the consequence of myelin disruption and does not require the adaptive immune system. Our data therefore indicate that protecting axons from the consequences of myelin damage is critical, and we believe that fostering efficient myelin clearance in concert with supporting remyelination is likely to be beneficial.

Currently available models for studying the consequences of disrupting myelinaxon interactions are based on genespecific mutations (Nave and Trapp, 2008),
A

|ba-1 / Niss|
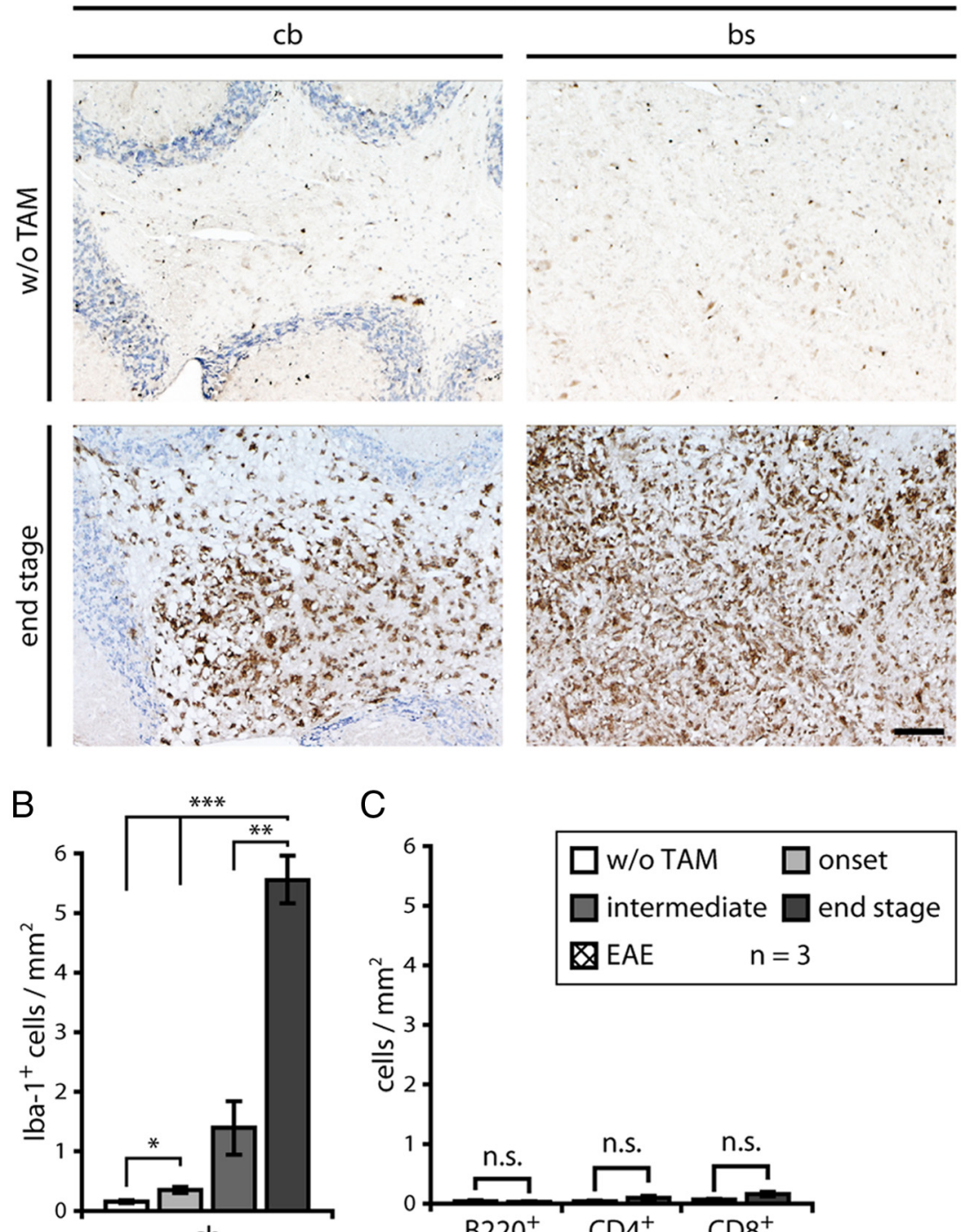

C
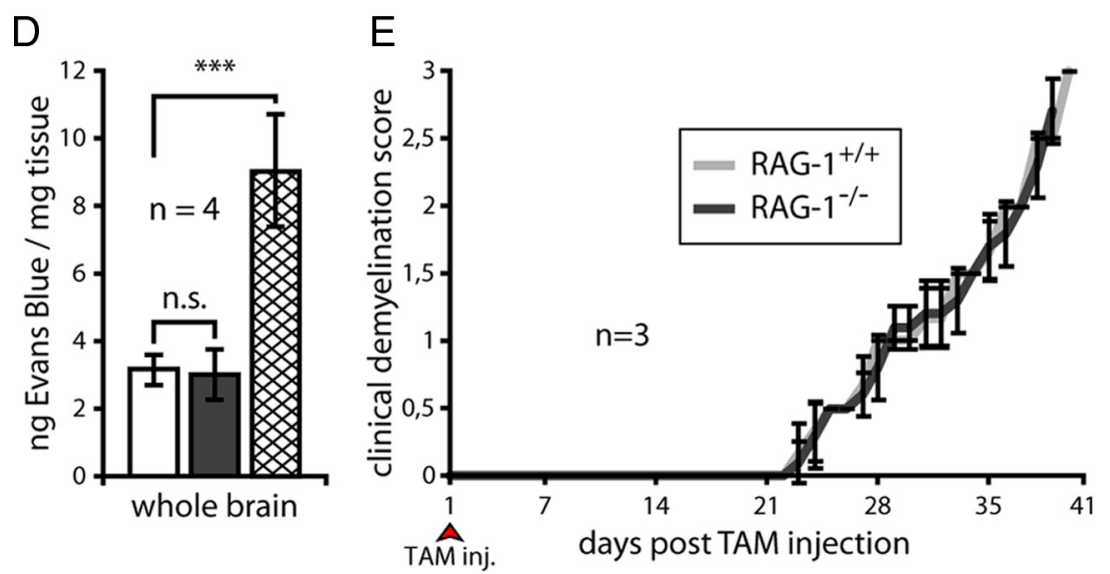

Figure 7. No detectable involvement of the adaptive immune system in disease development after genetically induced adult oligodendrocyte death. $\boldsymbol{A}$, Histological staining for activated monocytic cells (lba-1) reveals accumulation in damaged tissues of $c b$ and bs in experimental mice. $\boldsymbol{B}$, Quantifications of $\mathrm{Iba}-1^{+}$cells over the time course of disease development shows increasing accumulation of activated microglia in the severely affected cb. C, Quantifications of stainings for B220, CD4, and CD8 at end stage show no significant increase in B-and T-cells within the brain, suggesting no major adaptive immune response to adult oligodendrocyte death reaching the CNS. D, Evans blue penetration assay reveals no leakage of the blood-brain barrier in experimental animals at end stage, while prominent influx was found in the inflammatory EAE model. $\boldsymbol{E}$, When experimental mice are depleted of functional lymphocytes by breeding on a RAG-1-null background, symptomatic progression remains unchanged compared with the RAG-1 wild-type background. Data are given as mean \pm SEM. inj., Injection. ${ }^{*} p<0.05,{ }^{* *} p<0.01,{ }^{* * *} p<0.001$, Student's t test. Scale bars, $100 \mu \mathrm{m}$. 
induced autoimmunity against CNS components (Baxter, 2007), and the use of various toxins (Blakemore and Franklin, 2008). Each of these established models is valuable to study specific aspects of the underlying mechanisms of myelin pathology, but none allows the well defined and controlled specific ablation of adult OLGs in the intact mouse CNS. Importantly, in the model we describe here there are no confounding primary effects on other cell types, including on axons, which remain concerns with the use of toxins or immune-mediated models. After induction of genetic recombination and a comparable synchronized loss of OLGs in all areas examined, we observed a highly reproducible disease process with a relatively late onset and slow progression. The first clinical disease signs coincided with myelin pathology, including myelin vacuolation, which most likely occur due to the decay of structurally important myelin components (Baumann and Pham-Dinh, 2001). The occurrence of vacuoles in affected brain areas correlated with the increase in T2 values in the respective ROIs observed in MRI. Myelin vacuoles increased both in size and numbers with disease progression and were associated with microglia activation and astrogliosis. However, phagocytic removal of myelin was slow. This was most likely due to the $\mathrm{BBB}$ remaining intact. Resident microglia would therefore appear to be inefficient in removing myelin debris in the absence of bloodderived macrophages or stimulation by recruited adaptive immune cells (Neumann et al., 2009). Similar mechanisms have been suggested to underlie the protracted myelin clearance in Wallerian degeneration involving apoptotic oligodendrocyte death after optic nerve and spinal cord injury (Vargas and Barres, 2007).

Toxin-induced demyelination in the adult rodent CNS is characterized by brisk removal of myelin debris and efficient remyelination (Zhao et al., 2006). It was striking in our model that the poor removal of myelin debris was also associated with poor remyelination, a result consistent with earlier studies on the remyelination-inhibitory properties of uncleared myelin debris (Kotter et al., 2006; Baer et al., 2009). Although inhibitory to OPC differentiation, myelin debris does not effect OPC proliferation, which most likely accounts for the OPC proliferation that we observed in our model. Some limited remyelination was observed in the corpus callosum. This area of white matter contains almost exclusively small-diameter, thinly myelinated axons, so it is possible that the relatively small amount of debris generated in this region was insufficient to inhibit remyelination from occurring. By contrast, in the deep cerebellar white matter, which contains large heavily myelinated axons, the quantity of myelin debris is greater and may account for the lack of remyelination seen in this region. Significantly, it was in the areas in which remyelination was absent that the most severe axon pathology was observed. Thus, we favor the hypothesis that nonefficient myelin debris clearance, in combination with impaired remyelination, leads to axonal damage. This impairment is not dependent on the adaptive immune system since the extent of both, myelin alterations and axonal damage, was identical on an immune-deficient mouse background. Our findings are in line with the view that myelinating glia provide vital protective and/or nurturing support to axons (Nave and Trapp, 2008; Nave, 2010). Nonetheless, we cannot formally exclude direct damage contributions through microglia, astroglia, toxic effects of myelin debris, or combinations of all these factors.

Our mouse model is not characterized by extensive inflammation, nor does the immune system participate in regulating the extent of myelin vacuolation, activation of microglia, or the degree of axonal damage. Thus, we expect that this model will be valuable to study the consequences of adult OLG death on neuron-glia interactions and tissue integrity without the overshadowing influence by the adaptive immune system. In turn, the model allows the specific activation and modulation of regulatory immune and nonimmune pathways to examine, for example, how myelin clearance and potential detrimental consequences could be improved.

Although induction of adult OLG cell death cannot be considered to be a an accurate facsimile of all types of MS, it is noteworthy that the observed pathology bears much resemblance with described early MS lesions that are characterized by major OLG loss (Barnett and Prineas, 2004). Thus, these mice might be useful to follow up on the disease mechanism in these particular types of patients and lesions.

In summary, we present a highly reproducible animal model of induced adult OLG cell death that has ample potential for future studies such as axon-glia interactions, demyelination, mechanisms of myelin debris clearance, and remyelination.

\section{References}

Adornato B, Lampert P (1971) Status spongiosus of nervous tissue. Electron microscopic studies. Acta Neuropathol 19:271-289.

Baer AS, Syed YA, Kang SU, Mitteregger D, Vig R, Ffrench-Constant C, Franklin RJ, Altmann F, Lubec G, Kotter MR (2009) Myelin-mediated inhibition of oligodendrocyte precursor differentiation can be overcome by pharmacological modulation of Fyn-RhoA and protein kinase C signalling. Brain 132:465-481.

Barnett MH, Prineas JW (2004) Relapsing and remitting multiple sclerosis: pathology of the newly forming lesion. Ann Neurol 55:458-468.

Barnett MH, Sutton I (2006) The pathology of multiple sclerosis: a paradigm shift. Curr Opin Neurol 19:242-247.

Baumann N, Pham-Dinh D (2001) Biology of oligodendrocyte and myelin in the mammalian central nervous system. Physiol Rev 81:871-927.

Baxter AG (2007) The origin and application of experimental autoimmune encephalomyelitis. Nat Rev Immunol 7:904-912.

Blakemore WF, Franklin RJ (2008) Remyelination in experimental models of toxin-induced demyelination. Curr Top Microbiol Immunol 318:193-212.

Brockschnieder D, Pechmann Y, Sonnenberg-Riethmacher E, Riethmacher D (2006) An improved mouse line for Cre-induced cell ablation due to diphtheria toxin A, expressed from the Rosa26 locus. Genesis 44:322-327.

Compston A, Coles A (2008) Multiple sclerosis. Lancet 372:1502-1517.

Duncan ID, Brower A, Kondo Y, Curlee JF Jr, Schultz RD (2009) Extensive remyelination of the CNS leads to functional recovery. Proc Natl Acad Sci U S A 106:6832-6836.

Ellis E (2006) Corrected formulation for Spurr low viscosity embedding medium using the replacement epoxide ERL 4221. Microsc Microanal 12:288-289.

Franklin RJ, ffrench-Constant C (2008) Remyelination in the CNS: from biology to therapy. Nat Rev Neurosci 9:839-855.

Kotter MR, Li WW, Zhao C, Franklin RJ (2006) Myelin impairs CNS remyelination by inhibiting oligodendrocyte precursor cell differentiation. J Neurosci 26:328-332.

Kroner A, Ip CW, Thalhammer J, Nave KA, Martini R (2010) Ectopic T-cell specificity and absence of perforin and granzyme B alleviate neural damage in oligodendrocyte mutant mice. Am J Pathol 176:549-555.

Leone DP, Genoud S, Atanasoski S, Grausenburger R, Berger P, Metzger D, Macklin WB, Chambon P, Suter U (2003) Tamoxifen-inducible gliaspecific Cre mice for somatic mutagenesis in oligodendrocytes and Schwann cells. Mol Cell Neurosci 22:430-440.

Lucchinetti C, Brück W, Parisi J, Scheithauer B, Rodriguez M, Lassmann H (2000) Heterogeneity of multiple sclerosis lesions: implications for the pathogenesis of demyelination. Ann Neurol 47:707-717.

Martini R, Toyka KV (2004) Immune-mediated components of hereditary demyelinating neuropathies: lessons from animal models and patients. Lancet Neurol 3:457-465.

Matthaei I, Polman CH, de Groot CJ, Dijkstra CD, Koetsier JC, Sminia T (1989) Observer agreement in the assessment of clinical signs in experimental allergic encephalomyelitis. J Neuroimmunol 23:25-28.

Mendel I, Kerlero de Rosbo N, Ben-Nun A (1995) A myelin oligodendrocyte 
glycoprotein peptide induces typical chronic experimental autoimmune encephalomyelitis in H-2b mice: fine specificity and T cell receptor V beta expression of encephalitogenic T cells. Eur J Immunol 25:1951-1959.

Mombaerts P, Iacomini J, Johnson RS, Herrup K, Tonegawa S, Papaioannou VE (1992) RAG-1-deficient mice have no mature B and T lymphocytes. Cell 68:869-877.

Nave KA (2010) Myelination and the trophic support of long axons. Nat Rev Neurosci 11:275-283.

Nave KA, Trapp BD (2008) Axon-glial signaling and the glial support of axon function. Annu Rev Neurosci 31:535-561.

Neumann H, Kotter MR, Franklin RJ (2009) Debris clearance by microglia: an essential link between degeneration and regeneration. Brain 132: $288-295$.

Ogunshola OO, Djonov V, Staudt R, Vogel J, Gassmann M (2006) Chronic excessive erythrocytosis induces endothelial activation and damage in mouse brain. Am J Physiol Regul Integr Comp Physiol 290:R678-R684.

Rivers LE, Young KM, Rizzi M, Jamen F, Psachoulia K, Wade A, Kessaris N, Richardson WD (2008) PDGFRA/NG2 glia generate myelinating oligodendrocytes and piriform projection neurons in adult mice. Nat Neurosci 11:1392-1401.

Schmued LC, Stowers CC, Scallet AC, Xu L (2005) Fluoro-Jade C results in ultra high resolution and contrast labeling of degenerating neurons. Brain Res 1035:24-31.
Srinivas S, Watanabe T, Lin CS, William CM, Tanabe Y, Jessell TM, Costantini F (2001) Cre reporter strains produced by targeted insertion of EYFP and ECFP into the ROSA26 locus. BMC Dev Biol 1:4.

Stidworthy MF, Genoud S, Suter U, Mantei N, Franklin RJ (2003) Quantifying the early stages of remyelination following cuprizone-induced demyelination. Brain Pathol 13:329-339.

Suter U, Scherer SS (2003) Disease mechanisms in inherited neuropathies. Nat Rev Neurosci 4:714-726.

Takahashi K, Prinz M, Stagi M, Chechneva O, Neumann H (2007) TREM2transduced myeloid precursors mediate nervous tissue debris clearance and facilitate recovery in an animal model of multiple sclerosis. PLoS Med 4:e124.

Trapp BD, Nave KA (2008) Multiple sclerosis: an immune or neurodegenerative disorder? Annu Rev Neurosci 31:247-269.

Vargas ME, Barres BA (2007) Why is Wallerian degeneration in the CNS so slow? Annu Rev Neurosci 30:153-179.

Wegner M (2001) Expression of transcription factors during oligodendroglial development. Microsc Res Tech 52:746-752.

Zhao C, Li WW, Franklin RJ (2006) Differences in the early inflammatory responses to toxin-induced demyelination are associated with the age-related decline in CNS remyelination. Neurobiol Aging 27:12981307. 